\title{
CURE OF AN OLD DISLOCATION OF THE HUMERUS.
}

BY PROF. DIEFFENBACH.

Herr Th., a large land owner, upwards of thirty years old, had his right shoulder dislocated two years ago by a fall from his horse; the nature of the accident was not at first recognized, and afterwards, though all the usual means were adopted by several surgeons, the bone could not be returned to its place. The patient, therefore, came to Berlin: he was of a gaunt, powerful form, with a pale complexion and but little fat, and his muscles were strong and prominent under the skin. The injured right shoulder was an inch higher than the left; the acromion formed a sharp angle; on the outer side the shoulder was deeply hollowed, and the scapula lay flat. The right arm was thinner than the left, and stood out far from the body. The head of the humerus lay on the anterior side of the chest, close to the clavicle, and two inches from the upper portion of the sternum. The patient had a constant sensation of cold in the limb, and the creeping which he had formerly felt had ceased. The pulse in the right radial artery was rather weaker than in the left. The limb was useless, and only the hand could perform some slight actions.

By moving the arm in different directions, severe pain was produced in the part where the head lay surrounded by a thick wall of dense ligament into which it had worked itself. In drawing the arm outwards from the body, the pectoralis major, latissimus dorsi, teres major and teres minor became tense, with extreme pain. The last three of these muscles felt hard and tense, even when the arm was not drawn outwards. An attempt to reduce such a dislocation without dividing these muscles and the new joint would have been extremely dangerous, and had been found impossible; but (says the Professor) I anticipated success from the subcutaneous division of everything that resisted me.

The patient being placed on the table, with one folded sheet passed under the right axilla and held by six assistants, another fastened round the right hand and held by six more, and a third round the upper part of the humerus and held by three more (in the manner usually adopted by me in old luxations), the two first sets of assistants were ordered to pull against each other. I next bade them make a slowly-increased extension, and then stop; I then passed a small scythe-shaped knife through the skin, and divided the nost tense portion of the pectoralis major close to its tendon, which yielded with a cracking sound. I then again introduced the knife at the posterior border of the axilla, and divided one after the other the latissimus dorsi, the teres major, and the teres minor. All these muscles gave way with a cracking noise, which was increased by the resonance of the chest. I next passed my knife into three places by the head of the humerus, and divided in a similar manner under the skin, the dense and hard false ligaments which surrounded the new joint, and lessening the extension, I loosened the head by a few rotations.

A powerful extension was now again commenced on both sides, and the three assistants behind the patient pulled suddenly while I conducted 
the humerus towards the joint, into which it slipped on a sudden, without again springing out. One shoulder looked now just like the other. The thorax, the shoulder and the arm were enveloped with bandages which were soaked with paste, and after a few hours they all became dry and hard, and prevented any motion of the right side.

The bleeding from the wounds, which were not larger than those made in phlebotomy, was at most a few drops. No unpleasant symptoms ensued, and the patient suffered even less than the majority of persons in whom I bave reduced old dislocations. On the ninth day I took off the bandage; both shoulders had exactly the same level and form, and there was neither swelling nor pain. The punctures in the axilla had completely healed, and scarcely a trace of them could be found; there was no collection of blood or pus. The arm was, already capable of motion, and its actions were far less hindered than they are sometimes after the reduction of recent dislocations; because in them there is often for a long time a sensitive contraction of the unnaturally-stretched muscles, while in this case the division of the resisting muscles apd' of the newly-formed joint not only rendered the reduction possible, but at the same time diminished its after consequences. The limb is now again restored to perfect utility.

The Professor adds that he had lately occasion to reduce a luxation of the foot backwards, of upwards of a year's standing, by dividing the tendo-Achillis, which forcibly drew the heel upwards. This limb also became useful again.-Medicinische Zeitung.

\section{BOSTON MEDICAL AND SURGICAL JOURNAL.}

B O S T N, J U L $22,1840$.

\section{MEDICAL AND PHYSIOLOGICAL COMMENTARIES.}

Mention was made, last week, of this voluminous work, by Martyn Paine, M.D., of New York; but the mass of matter was altogether too formidable for any person to read in the short time of seven or eight days, interrupted very frequently by other concerns. Even now it is difficult to do more than enumerate the titles of some sections, which are resting places or subdivisions of arguments. Volume first begins with the vital powers. "Life," says Dr. Paine, "philosophically considered, is a cause, and productive of results which constitute life in a popular sense." This proposition is closed by an appendix - theories of galvanism. Afterwards comes the philosophy of the operation of the loss of blood, followed by appendix No. 1 and 2. Section third treats of the humoral pathology, subdivided into fourteen sections, with appendix 1,2 and 3 . When the circumstance of there being 715 pages, royal octavo, in this one volume, is recollected, it need hardly be said that each topic is discussed through all the ramificasecond volume already been asked if his is a book that will be useful to physicians, we beg leave to waive the question for after consideration. No further evi- 